Original Article

\title{
Incidence of Central Diabetes Insipidus among the Patients Undergoing Pituitary Tumor Surgery through Trans-sphenoidal Approach
}

\author{
*Ialam KMT', Alam S', Amin R', Deb-nath $H^{4}$, Hossain $M^{5}$, Khan $A H^{1}$, Hossain ATMM², Barua KK
}

\begin{abstract}
This study was carried out to find out the incidence of central diabetes insipidus (DI) among the patients undergoing pituitary tumor surgery through trans-sphenoidal approach either endoscopic or microsurgical for the first time. Patients with central (Neurogenic) diabetes insipidus prior to surgery, comorbidities like diabetes mellitus, kidney diseases, electrolyte imbalance, recurrent cases were excluded from this study. Patients were followed up to 7 th postoperative day by recording and analyzing findings of postoperative serum electrolytes, urinary specific gravity, hourly urinary volume for establishing diabetes insipidus. $76.9 \%$ and $23.1 \%$ of patients developed diabetes insipidus those who underwent pituitary tumor surgery by trans-sphenoidal endoscopic approach and microsurgical approach respectively. $70 \%$ and $30 \%$ of patients did not develop diabetes insipidus those who underwent pituitary tumor surgery by trans-sphenoidal endoscopic and microsurgical approach respectively. There was no significant deference between the approaches were found. Fisher exact test was done and p-value was 0.712. Prediction of DI help us in pre-operative counseling and post-operative management of the patients as well as to reduce complications related morbidity after pituitary tumor surgery.
\end{abstract}

1. *Dr. K.M. Tarikul Islam, Assistant Professor Department of Neurosurgery, Bangabandhu Sheikh Mujib Medical University (BSMMU), Dhaka. E-mail: dr.tarik2007@yahoo.com

2. Shamsul Alam, Assistant Professor, Department of Neurosurgery BSMMU, Dhaka.

3. Dr. Rezaul Amin, Assistant Professor, Department of Neurosurgery, BSMMU, Dhaka.

4. Dr. Haradhon Deb-nath, Associate Professor, Department of Neurosurgery, BSMMU, Dhaka.

5. Dr. Mohammad Hossain, Associate Professor, Department of Neurosurgery, BSMMU, Dhaka.

6. Dr. Akhlaque Hossain Khan, Associate Professor, Department of Neurosurgery, BSMMU, Dhaka.

7. Dr. ATM Mosharef Hossain, Professor, Department of Neurosurgery BSMMU, Dhaka.

8. Prof. Kanak Kanti Barua, Professor \& Chairman Department of Neurosurgery, BSMMU, Dhaka.

*For Correspondence
Key words: Diabetes insipidus, Trans-sphenoidal approach, pituitary tumor.

\section{INTRODUCTION}

The pituitary gland is a remarkable organ that is located at the base of the brain at the center of the skull base. The gland controls an array of endocrine functions important for human growth and metabolism. The most common pituitary- related pathologies that are surgically treated are pituitary tumors (mostly adenomas) and pituitary apoplexy. The classic indications for surgical treatment include visual loss from progressive mass effect, hormonal control in hyper functioning adenomas, failure of medical therapy, and necessity for a tissue diagnosis. Transcranial approaches to the sella for treating pituitary pathologies have been in existence since the late 19th century. In the early 1900 s, Hirsch descried a trans nasal approach to the sella turcica that was refined by Gushing, who standardized the trans labial, transseptal, Trans-sphenoidal approach to the pituitary gland. Recently, given the technologic enhancements in endoscopic visualization and instrumentation, the endo-nasal endoscopic Trans-sphenoidal approach has gained popularity for addressing pathology of the pituitary gland. ${ }^{1}$ Diabetes insipidus (DI) is a common complication following pituitary surgery. This condition can be transient or permanent and the signs and symptoms of this disorder can be mimicked by the normal postoperative course. ${ }^{2}$

There are two subtypes of DI: nephrogenic and central (neurogenic). Nephrogenic DI occurs when there is an inadequate response to AVP in the renal tubules, leading to an inability to concentrate urine; this can be caused by certain drugs, hypercalcemia, and primary kidney diseases. ${ }^{3}$ Central (neurogenic) diabetes insipidus (DI) is a significant postoperative complication of pituitary tumor resection. Disturbance of the posterior pituitary, pituitary stalk, or neurons originating in the Paraventricular or supraoptic hypothalamic nuclei during pituitary tumor resection may lead to transient or permanent imbalance of anti-diuretic hormone regulated water homeostasis. Clinical manifestations consist of polydipsia and uncontrolled excretion of dilute urine, which, if not treated, lead to elevated serum sodium and serum osmolality. ${ }^{4}$ 
Most cases of DI after pituitary surgery have been reported as transient, resolving within the first few days postoperatively. Nevertheless, studies report permanent DI ranging from $0.5 \%$ which constitutes a noteworthy risk 5 . The overall incidence of any postoperative (transient or permanent) DI in trans-sphenoidal pituitary surgery series has been reported to range from 1.6 to $31 \% \%^{6,7,8}$. Recently, Nemergut et al. reported their large series of patients undergoing non-endoscopic Trans-sphenoidal pituitary microsurgery?.

Polyuria is common after trans-sphenoidal surgery; however, it is not always due to DI. These should be considered and excluded before treatment of DI is initiated. Acromegalic patients have been known to have increased urinary output following resection of the pituitary microadenoma due to diuresis of excess fluid in the soft tissues. Nevertheless, polyuria remains a hallmark of DI. As such, accurate measurement of urine output is critical. When DI is suspected, additional tests are needed to confirm the diagnosis including measurement of urine specific gravity, urineand serum osmolality, and serum sodium. A diagnosis of DI is contingent upon the presence of polyuria and polydipsia in conjunction with specific laboratory abnormalities ${ }^{2}$.

Unfortunately, there are a wide range of measurements that have been used to establish a diagnosis of DI in the literature. For example, various authors have reported different thresholds of elevated urine output that should raise suspicion for DI such as $>250-500 \mathrm{ml} / \mathrm{hr}$ for 3 consecutive hours and $2.5-18 \mathrm{~L} /$ day. Urine specific gravity $<1.005$ is often used as a diagnostic parameter of DI11. Urine osmolality $<300 \mathrm{mOsm} / \mathrm{Kg}$ and serum osmolality $>300 \mathrm{mOsm} / \mathrm{kg}$ are also thought to be diagnostic of DI. In addition, one should be suspicious of DI when serum sodium increases to levels $>140-145$ mequ/L10, ${ }^{11}$.

Transient DI is commonly seen after Trans-sphenoidal pituitary surgery. With a transnasal microsurgical approach, the rate of transient DI has been reported between 1.6 and $45.6 \%{ }^{11}$. The incidence of temporary DI following a transnasal endoscopic approach has been reported between 2.5 and $15.2 \%{ }^{12}$. Similar studies have shown that the rate of DI is roughly the same between transnasal microscopic resection and transnasal endoscopic resection ${ }^{13}$.

However, a recent meta-analysis by Goudakos et al. found that postsurgical DI was less frequent in those who underwent endoscopic surgery compared to those who had microsurgical resection $(15 \% \text { vs. } 28 \%, p=0.03)^{2}$.
Diabetes insipidus is a common but usually transient complication following pituitary surgery. In rare instances of massive damage to AVP-producing magnocellular neurons of the hypothalamus, a permanent lack of endogenous vasopressin ensues. While certain factors appear to carry a higher risk for postoperative DI, it is important to monitor all postsurgical patients closely in an intensive care setting and to treat DI when appropriate. Other causes of postoperative polyuria must be ruled out, so as to avoid unnecessary pharmacotherapy. Meticulous surgical technique and careful preservation of the critical neurovascular structures in the hypothalamic-pituitary axis are essential in averting postsurgical $\mathrm{D}^{\mathrm{I}}$.

\section{MATERIALS AND METHODS}

This cross sectional type of observational study was carried out in the department of Neurosurgery, Bangabandhu Sheikh Mujib Medical University July, 2013 to June, 2014 on 33 consecutive patients with age ranged from 24 to 70 years. Among 33 Patients, 20 ware male. All the consecutive patients who underwent surgical intervention for pituitary tumor for the first time through Trans-sphenoidal approached either endoscopic or microsurgical were included in this study. Patients with central (Neurogenic) diabetes insipidus prior to surgery, co-morbidities like diabetes mellitus, kidney diseases, electrolytes imbalance, recurrent cases were excluded from this study. All patients were evaluated by detailed history, thorough general and neurological examination. Patients were followed up to 7 th postoperative day. Findings of postoperative serum electrolytes, urinary specific gravity and hourly urinary volume were recorded and analyzed for establishing diabetes insipidus. Data collection sheet was used to collect necessary information. Informed written consent was taken from each participant or guardian before data collection. Data was processed by utilizing IBM SPSS statistical program (Version 17.0). Results were described in frequencies or percentage. Statistical comparison was done using fisher exact test, and chi-square test. P-value $\leq .05$ was considered statistically significant.

\section{RESULTS}

The age range was from 24 to 70 years. Mean age of the patients was $40.5 \quad 1 \pm 10.58$ years. Maximum patients $(73.4 \%)$ were in between 21 to 50 years. Most patients were male with a male female ratio was $1.53: 1$. Among 33 patients $57.6 \%$ had normal level of serum sodium ion concentration and $42.4 \%$ of the patient had hypernatremia in postoperative period. $23.1 \%$ developed diabetes insipidus were $<30$ years of age, $38.5 \%$ of patients were between 
$31-40$ years age group and $38.5 \%$ of patients were $>40$ years age group. The patients who did not develop diabetes insipidus $15.0 \%$ were $<30$ years age group, $50.0 \%$ of patients were between $31-40$ years age group and $>40$ age group of patients were $35.0 \%$. 39.5\% of male and $60.5 \%$ of female developed diabetes insipidus. Among the patients who did not develop diabetes insipidus $75.0 \%$ of patients were male and $25 \%$ of patients were female. Most of the patients $(92.9 \%)$ having $\mathrm{Na}+$ concentration $>145 \mathrm{mmol} / \mathrm{L}$ had DI.

$36.4 \%$ of patients had tumor size (maximum diameter in coronal or sagittal plan of MRI) were $<30 \mathrm{~mm}$ and in $63.6 \%$ of patient's tumor size were. $>30 \mathrm{~mm}$. The range of tumor diameter was between $15 \mathrm{~mm}$ and $50 \mathrm{~mm}$. (Table-I)

Table I: Distribution of patients according to pre-operative image finding (diameter of tumor) $(n=33)$

\begin{tabular}{lccc}
\hline Tumor diameter $(\mathrm{mm})$ & $\mathrm{N}(\%)$ & Mean \pm SD & Mim-Max \\
\hline$<30$ & $12(36.4)$ & $21.91 \pm 4.10$ & $15-25$ \\
$>30$ & $21(63.6)$ & $35.28 \pm 5.36$ & $30-50$ \\
Total & $33(100.00)$ & $30.42 \pm 8.15$ & $15-50$ \\
\hline
\end{tabular}

Table II shows that resection through Trans-sphenoidal endoscopic rout was done on $72.7 \%$ of patients. $27.3 \%$ of patients underwent surgery through Trans-sphenoidal microsurgical approach.

Table II: Distribution of patients according to operative procedure $(n=33)$

\begin{tabular}{lcc}
\hline Pre-operative image finding & Frequency & Percentage \\
\hline Trans-sphenoidal endoscopic & 24 & $72.7 \%$ \\
Trans-sphenoidal microsurgical Approach & 9 & 27.3 \\
Total & 33 & 100.0 \\
\hline
\end{tabular}

Table III shows that among 33 patients $57.6 \%$ had normal level of serum sodium ion concentration and $42.4 \%$ of the patient had hypernatremia in postoperative period.

Table III: Distribution of patients according to increased and normal $\mathrm{Na}+$ concentration $(n=33)$

\begin{tabular}{lcc}
\hline Normal $\mathrm{Na}+$ concentration & Frequency & Percentage \\
\hline$<145$ & 19 & $57.6 \%$ \\
$>145$ & 14 & 42.4 \\
Total & 33 & 100.0 \\
\hline
\end{tabular}

$33.4 \%$ cases developed diabetes insipidus in postoperative period and $60.6 \%$ case did not develop diabetes insipidus. (Table IV)

Table IV: Distribution of patients according to development of diabetes insipidus (D1) $(n=33)$.

\begin{tabular}{lcc}
\hline Development of DI & Frequency & Percentage \\
\hline Absent & 20 & 60.6 \\
Present & 13 & 39.4 \\
Total & 33 & 100.0 \\
\hline
\end{tabular}

Table $\mathrm{V}$ shows that among the patients who developed diabetes insipidus $(n=13)$ of them $76.9 \%$ developed in first postoperative day and $23.0 \%$ developed in the second post-operative day.

Table V: Distribution of patients according to postoperative day of development of diabetes insipidus (Dl) $(\mathrm{n}=13)$.

\begin{tabular}{lcc}
\hline Postoperative Day of development of DI & Frequency & Percentage \\
\hline First & 10 & 76.9 \\
Second & 3 & 23.00 \\
\hline
\end{tabular}

Among $<30 \mathrm{~mm}$ tumor diameter group $30.8 \%$ of patients developed diabetes insipidus and $40.0 \%$ of patients did not develop diabetes insipidus. Among $>30 \mathrm{~mm}$ tumor diameter group $69.2 \%$ of patients developed diabetes insipidus and $60.0 \%$ of patients did not develop diabetes insipidus.

Table VI: Development of diabetes insipidus (DI) in tumor size groups $(n=33)$.

\begin{tabular}{lccc}
\hline $\begin{array}{l}\text { Tumor } \\
(\mathrm{mm})\end{array}$ & \multicolumn{3}{c}{ Diabetes insipidus $(\mathrm{Dl})$} \\
\cline { 2 - 4 } P value & \multicolumn{2}{c}{ Present $\mathrm{n}(\%)$} & Absent $\mathrm{n}(\%)$ \\
\hline$<30$ & $4(30.8)$ & $8(40.0)$ \\
$>30$ & $9(69.2)$ & $12(60.0)$ & \\
Total & $13(100.0)$ & $20(100.0)$ & $(100.0)$ \\
& \multicolumn{3}{c}{0.590} \\
\hline
\end{tabular}

There was no significant deference between the two groups and $\mathrm{p}$-value was 0.590 . (Table VI). 
Table VII: Development of diabetes insipidus (DI) in operative procedures

\begin{tabular}{lccc}
\hline $\begin{array}{l}\text { Tumor } \\
\text { (mm) }\end{array}$ & \multicolumn{3}{c}{ Diabetes insipidus (Dl) } \\
\cline { 2 - 4 } P value & \multicolumn{2}{c}{ Present n(\%) } & Absent n (\%) \\
\hline $\begin{array}{l}\text { Trans-sphenoidal } \\
\text { Approach }\end{array}$ & Endoscopic & $10(76.9)$ & $14(70.00)$ \\
$\begin{array}{l}\text { Trans-sphenoidal } \\
\text { Approach }\end{array}$ & Microsurgical & $3(23.1)$ & $6(30.0)$ \\
Total & $13(100.0)$ & $20(100.0)$ & 0.712 \\
\hline
\end{tabular}

$76.9 \%$ of patients developed diabetes insipidus and $70.0 \%$ of patients did not develop diabetes insipidus those who underwent pituitary tumor surgery by trans-sphenoidal endoscopic approach; $23.1 \%$ of patients developed diabetes insipidus and $30.0 \%$ of patients did not develop diabetes insipidus those who underwent pituitary tumor surgery by trans-sphenoidal microsurgical approach. There was no significant deference between the approaches were found. Fisher exact test was done and p-value was 0.712. (Table VII)

\section{DISCUSSION}

Incidence of DI in postoperative patients in our study is $39.4 \%$ which is similar to other study and DI occurred in $86.3 \%$ of the affected patient on the second postoperative day $^{11}$. It was found that $71.2 \%$ of the patients manifested DI within 24 hours of undergoing surgical intervention ${ }^{14}$. In our study we found that $76.9 \%$ of the patients developed DI on the first postoperative day and $23.0 \%$ of the patients developed DI on the second postoperative day. Our observation is in agreement with reports of postoperative DI generally occurring within 24 hours of the surgical procedure.

The mean age of our study population was 40.51 years ( $\mathrm{SD} \pm 10.58$ years) which is similar to other study were mean age at the time of surgery was $40.1,{ }^{14}$.

Adams JR. et al. included total 59 patients with DI. Of them 16 were men and 43 were women ${ }^{14}$. They found that $27.1 \%$ of men and $72.9 \%$ of women had postoperative DI. We found $39.5 \%$ men and $60.5 \%$ women had postoperative DI which is nearly similar to the finding above.

It was found that DI is commonly seen after trans-sphenoidal pituitary surgery. With transnasal microsurgical approach the rate of development of DI ranges between $1.6 \%$ and $45.6 \%$. In our study $23.1 \%$ of patients develop DI who underwent trans-sphenoidal microsurgical approach for surgical removal of pituitary macroadenoma which was within range according to above studies $^{11}$

It was found that the incidence of DI following a transnasal endoscopic approach between $2.5 \%$ and $15.2 \%$. In our observation we found that $76.9 \%$ of the patients developed DI who underwent pituitary tumor resection by trans-sphenoidal endoscopic approach. Our incidence of DI is much more than that of Jho HD'S findings ${ }^{12}$.

It was shown that the relationship between elevated serum sodium levels $(>145 \mathrm{mmol} / \mathrm{L})$ within the first five days postoperatively and the incidence of overall postoperative $\mathrm{D}^{\mathrm{I}}$. They found that the development of hypernatremia within five days postoperatively was strongly associated with postoperative DI (transient or permanent) $(\mathrm{p}=<\mathrm{O} .0001)$. Of the 14 patients who had a serum sodium level $>145 \mathrm{mmol} / \mathrm{L}$ within the first five days postoperatively, all 14 met criteria for DI. In our study among the 33 patients 14 of them developed hypernatremia (serum sodium level $>145 \mathrm{mmol} / \mathrm{L}$ ) within first two days postoperatively and 13 of them met the criteria for DI. So, in our study we also found that there was a strong association between postoperative hypernatremia with DI. But hypernatremia of another patient due to over infusion of sodium containing fluid.

Incidence of development of serum sodium imbalance in both male and female is equal and the ratio is 1.1:0.9 $9^{15}$. Previous study also found no impact of gender on the incidence of postoperative $\mathrm{D}^{\mathrm{I7}}$. In our study there was association between gender and postoperative DI $(\mathrm{p}=0.035)$ which does not correspond with the study of the above mentioned investigator.

Some authors have shown that DI is more common after the resection of macroadenoma. The circumstance is likely to be caused by the more aggressive gland and stalk manipulation required during resection as well as changes in sellar and suprasellar anatomy in patients with macroadenoma? .

It was found in their that among the patients who had a pituitary adenoma and no preoperative DI, $69.4 \%$ had macroadenomas. Microadenomas were found in $30.6 \%$ of patients ${ }^{9}$. Diabetes insipidus was more likely to be diagnosed in patients with microadenomas $(21.6 \%$ developed postoperative DI) than with macroadenomas (14.3\% developed postoperative DI) $(\mathrm{p}=0.049)$. They found that adenoma size had no impact on the development of persistent DI. In our study all the 33 patients had macroadenoma. Among them $63.6 \%$ had tumor diameter 
$>30 \mathrm{~mm}$ and $36.4 \%$ had tumor diameter $<30 \mathrm{~mm}$. In $>30 \mathrm{~mm}$ tumor diameter group $69.2 \%$ developed postoperative DI and $<30 \mathrm{~mm}$ tumor diameter group $38.8 \%$ developed postoperative DI $(\mathrm{p}=0.590)$. It was statistically insignificant. So, we could predict that large tumor sizehas no impact on the development DI. But we could not compare with microadenomas because in the perspective of our country we only found macroadenomas.

Trans-sphenoidal surgery, include more than $95 \%$ of the surgical indications in the sellar area and approximately 96\% of all pituitary adenomas (Jho et al., 2012). However, a recent meta-analysis by Goudakos et al. found that postsurgical DI was less frequent in those who underwent endoscopic surgery compared to those who had microsurgical resection ( $15 \%$ vs. $28 \%, p=0.03)^{17}$. In our study approach did not influence much the postoperative diabetes insipidus $(\mathrm{p}=0.0712)$ which does not correspond with meta-analysis of Goudakos et $\mathrm{a}^{16}$.

Limitation of the study were duration was short, limited number of patients, surgical procedures were performed by different surgeons, we could not find the incidence of postoperative DI in relation to the extent of tumor resection due to unavailability of postoperative imaging. So it remained unpredicted.

Early prediction of this notorious complication is essential for post-operative management of the patient. This will help us in preoperative counseling and postoperative management of the patients as well as to reduce complications related morbidity after the pituitary tumor surgery. Practitioners should prevent the overtreatment of polyuria with desmopressin, especially in patients who can keep up with urinary loses through oral fluid intake. Excessive desmopressin treatment can result in hyponatremia and significant morbidity.

\section{REFERENCES}

1. Singh A, Roth J, Anand VK \& Schwart TH. Anatomy of the Pituitary Gland and Para sellar Region. Endoscopic Pituitary Surgery. 2012:9

2. Scheckinger M, Szerlip R, Mittal S. Diabetes insipidus following resection of pituitary Tumors. Clinical Neurology and Neurosurgery. 2013; 115: 121-26.

3. Earley LE and Orloff J. The mechanism of anti-diuresis associated with the administration of hydrochlorothiazide to patients with vasopressin-resistant diabetes insipidus. Journal of Clinical Investigation. 1962: 1988-97.
4. Sigounas DG, Sharpless JL, Cheng DM, Johnson TG, Senior BA \& Ewend MG. Predictors and incidence of central diabetes insipidus after endoscopic pituitary surgery.Journal of Neurosurgery. 2008; 62:71 -8.

5. Ciric I, Ragin A, Baumgartner C, Pierce D. Complications of trans-sphenoidal surgery: Results of anational survey, review of the literature, and personal experience. Journal of Neurosurgery. 1997; 40: 225-37.

6. Black PM, Zervas NT, Candia GL. Incidence and management of complications of trans-sphenoidal operation for pituitary adenomas. Journal ofNeurosurgery. 1987; 20: 920-24.

7. Hensen J, Henig A, Fahlbusch R, Meyer M, Boehnert M, Buchfelder M. Prevalence, predictors and patterns of postoperative polyuria and hyponatremia in the immediate course after trans-sphenoidal surgery for pituitary adenomas. Clin Endocrinol (Oxf). 1999; 50: 431-39.

8. Singer PA and SevillaLJ. Postoperative endocrine management of pituitary tumors. Neurosurg Clin N Am.2003; 14: 123-38.

9. Nemergut EC, Zuo Z, Jane JA Jr, Laws ER Jr. Predictors of diabetes insipidus after Trans-sphenoidal surgery: A review of 881 patients. J Neurosurg. 2005; 103: 448-54.

10. Bajpai A, Kabra M, Menon PS. Central diabetes insipidus: clinical profile and factors indicating organic etiology in children. Indian Pediatrics. 2008; 45: 463-68.

11. Kristof RA, Rother M, Neuloh G, Klingmuller D. Incidence, clinical manifestations, and course of water and electrolyte metabolism disturbances following Trans-sphenoidal pituitary adenoma surgery: a prospective observational study. Journal ofNeurosurgery. 2009; 11: 555-62.

12. Jho HD. Endoscopic Trans-sphenoidal surgery. Journal of Neuro-Oncology. 2001; 54: 87-95.

13. Dumont AS, Nemergut EC, Jane Jr. JA, Laws Jr. ER. Postoperative care following pituitary surgery. Journal of Intensive Care Medicine.2005; 20: 127-40.

14. Adams JR, Blevins LS JR, Allen GS, Verity DK \& Devin JK. Disorders of water metabolism following Trans-sphenoidal pituitary surgery: a single institution's experience. Pituitary. 2006; 9: 93-9.

15. Staiger RD, Sarnthein J, Wiesli P, Schmid C \& Bernays RL. Prognostic factors for impaired plasma sodium homeostasis after Trans-sphenoidal surgery. $\mathrm{Br}$ JNeurosurg.2013; 27: 63-8. 
16. Goudakos JK, Markou KD \& Georgalas C. Endoscopic versus microscopic Trans-sphenoidal pituitary surgery: a systematic review and meta-analysis. Clinical Otolaryngology. 2011; 36: 212-20. 